\title{
Muscle Strength, Physical Activity, and Functional Limitations in Older Adults with Central Obesity
}

\author{
Cassandra M. Germain, ${ }^{1}$ John A. Batsis, ${ }^{2}$ Elizabeth Vasquez, ${ }^{3}$ and Douglas R. McQuoid ${ }^{1}$ \\ ${ }^{1}$ Department of Psychiatry and Behavioral Sciences, Duke University Medical Center, P.O. Box 3119, Durham, NC 27710, USA \\ ${ }^{2}$ Department of Medicine, Geisel School of Medicine at Dartmouth, Dartmouth-Hitchcock Medical Center, \\ 1 Medical Center Drive, Lebanon, NH 03756, USA \\ ${ }^{3}$ Department of Epidemiology and Biostatistics, School of Public Health, University at Albany (SUNY), One University Place, \\ Albany, NY 12203, USA
}

Correspondence should be addressed to Cassandra M. Germain; cassandra.germain@duke.edu

Received 9 October 2015; Revised 28 January 2016; Accepted 31 January 2016

Academic Editor: F. R. Ferraro

Copyright (C) 2016 Cassandra M. Germain et al. This is an open access article distributed under the Creative Commons Attribution License, which permits unrestricted use, distribution, and reproduction in any medium, provided the original work is properly cited.

\begin{abstract}
Background. Obesity and muscle weakness are independently associated with increased risk of physical and functional impairment in older adults. It is unknown whether physical activity (PA) and muscle strength combined provide added protection against functional impairment. This study examines the association between muscle strength, PA, and functional outcomes in older adults with central obesity. Methods. Prevalence and odds of physical (PL), ADL, and IADL limitation were calculated for 6,388 community dwelling adults aged $\geq 60$ with central obesity. Individuals were stratified by sex-specific hand grip tertiles and PA. Logistic models were adjusted for age, education, comorbidities, and body-mass index and weighted. Results. Overall prevalence of PL and ADL and IADL limitations were progressively lower by grip category. Within grip categories, prevalence was lower for individuals who were active than those who were inactive. Adjusted models showed significantly lower odds of PL OR 0.42 [0.31, 0.56]; ADL OR $0.60[0.43,0.84]$, and IADL OR $0.46[0.35,0.61]$ for those in the highest grip strength category as compared to those in the lowest grip category. Conclusion. Improving grip strength in obese elders who are not able to engage in traditional exercise is important for reducing odds of physical and functional impairment.
\end{abstract}

\section{Introduction}

Among those aged 60 and older, the combined prevalence of overweight and obesity, as assessed by body mass index (BMI), is estimated at over $70 \%$ [1]. The prevalence of central obesity is also rising among older adults in the US $[2,3]$ and is independently associated with functional impairment and disability $[4,5]$. Central obesity is also associated with muscle weakness [6,7], a known independent risk factor for physical disability [8] and mortality in older adults [9]. Thus, adults who carry excess weight and exhibit muscle weakness have a synergistic increased risk of functional impairment and subsequent disability than those who exhibit either symptom alone $[10,11]$.

Physical activity (PA) is associated with decreased risk of disability, delayed onset of functional impairment $[12,13]$, and recovery of function in older adults [14]. Physical activity is also associated with performance on key measures of physical performance including grip strength $[15,16]$. Our group has previously demonstrated that PA moderates the risk of physical and functional limitations in normal weight and obese older adults $[4,17,18]$. Although previous work has demonstrated the association between grip strength and disability in middle and older adults $[19,20]$, whether higher levels of PA coupled with greater grip strength provides added benefits against physical and functional limitations in older adults who are obese is unknown. The current study extends our previous work to investigate the independent and combined effects of grip strength and physical activity on physical and functional outcomes in older adults with central adiposity. We examined (a) prevalence of physical and functional limitations by grip strength, (b) whether having 
higher grip strength was associated with reduced odds of physical and functional limitations, and (c) whether grip strength combined with PA is associated with additional reductions in physical and functional limitations. Given the compounded risk of disability among obese elders who also exhibit muscle weakness, it is important to identify ways of improving physical and functional outcomes within this particularly vulnerable group.

\section{Methods}

2.1. Data Source. This current study is a secondary analysis of existing data obtained from the 2006 and 2008 waves of the enhanced face-to-face interview of the Health and Retirement Study (HRS). HRS is a nationally representative panel survey of community dwelling adults aged 50 and older conducted by the University of Michigan and supported by the National Institute on Aging. The initial HRS sample was drawn in 1992 from a multistage, clustered area probability design of households, which targeted individuals born between 1931 and 1941. Follow-up interviews and new cohort additions have occurred at regular intervals and have resulted in a nationally representative sample of American adults over the age of 50. A random one-half of the larger HRS sample was preselected to complete an enhanced face-to-face interview in 2006, which included physical and biomarker measurements. The second half of the HRS sample received the enhanced face-to-face interview in 2008. Inclusion of both 2006 and 2008 waves ensures that all HRS respondents who were eligible for and consented to the enhanced faceto-face interviews are included in the analyses. Additional descriptions of sampling procedures and study design are available online at http://hrsonline.isr.umich.edu/.

2.2. Sample. A total of 6,555 community dwelling adults aged 60 years and older with clinically elevated waist circumference (WC $\geq 88 \mathrm{~cm}$ for women; WC $\geq 102 \mathrm{~cm}$ for men) [ 21 , 22] and handgrip strength measurements were included in the sample. After eliminating respondents missing handgrip $(n=163)$ physical activity $(n=4)$, a total of $6,388(2,364$ men; 4,024 women) remained for analyses. Respondents of all races/ethnicities were included in the sample. Characteristics of those missing grip strength are provided in the Appendix. The study was exempted from Institutional Review Board review at all respective institutions due to the deidentified nature of the data used.

\subsection{Measures}

2.3.1. Muscle/Grip Strength. Participants' handgrip strength was measured using Smedley spring-type hand dynamometer $\left(\mathrm{TTM}^{\circledR}\right.$, Tokyo, Japan) in a standing position with their arm at their side at a 90-degree angle. Two measurements were taken with each hand (dominant and nondominant) and averaged across trials [23]. The highest averaged score from either hand was used as primary predictor in the current analyses. Sexspecific tertiles were determined using univariate analyses.
Participants were then classified into three categories by sexspecific grip tertiles: low grip (LG), mid grip (MG), and high grp (HG).

2.3.2. Physical Activity. Physical activity (PA) was assessed using a self-report physical health questionnaire as part of the HRS interview. Respondents were asked "How often do you take part in sports or activities that are vigorous, such as running or jogging, swimming, cycling, aerobics or gym, workout, tennis, or digging with a spade or shovel: more than once a week, once a week, one to three times a month, or hardly ever or never?" and "And how often do you take part in sports or activities that are moderately energetic such as, gardening, cleaning the car, walking at a moderate pace, dancing, floor or stretching exercises. ..?" Frequency of engagement in HRS was reported as follows: more than once a week, once a week, one to three times a month, or hardly ever or never. Respondents reporting that they engaged in moderate or vigorous PA at least once per week were classified as active; those reporting activity less than once per week were classified as inactive (ref $=0)$.

2.3.3. Physical Limitations (PL). Physical limitations (PL) in HRS were assessed using a self-reported questionnaire. Respondents were asked to report whether they had difficulties or were unable to (yes/no) perform the following tasks because of a health or physical problem: walking several blocks, walking 1 block, sitting 2 hours, getting up from chair, climbing one flight of stairs, stooping, reaching arms, pulling/pushing large objects, lifting weights, and picking up a dime. All yes responses were compiled into a summary score in the HRS database ranging from 0 to 10 . Univariate analyses revealed that $67 \%$ of our sample reported at least 1 limitation. For purposes of these analyses, respondents reporting difficulty or inability with performing two or more of the above tasks were classified as having physical limitations $(0=$ no limitations, $1=$ limitation $)$.

2.3.4. Functional Limitations. Activities of daily living (ADLs) and instrumental activities of daily living (IADLs) were used to assess functional limitations [24]. Respondents were classified as having ADL limitations if they reported difficulty or inability with one or more of the following: dressing, eating, or getting out of bed. Respondents were classified as having IADL limitations if they reported difficulty or inability with at least one of the following IADLs: preparing meals, managing money, or needing help with house/yard work. All limitations reported in this paper were measured in HRS using self-report. Respondents were instructed to exclude any difficulties that were expected to last less than three months.

\section{Analyses}

Overall prevalence and odds of physical, ADL, and IADL limitations was examined by grip strength category. Prevalence and odds of limitations were also examined within each grip category by activity level. Multivariate logistic 
TABLE 1: Sample characteristics.

\begin{tabular}{|c|c|}
\hline$N$ & 6,388 \\
\hline & $\mathrm{M}(\mathrm{SD})$ \\
\hline Age, years & $71.4(7.48)$ \\
\hline Education, years & $12.2(3.13)$ \\
\hline Comorbidities & $2.2(1.21)$ \\
\hline Waist circumference $(\mathrm{cm})$ & $108.0(12.50)$ \\
\hline Handgrip strength & $28.6(10.43)$ \\
\hline Body mass index $(\mathrm{kg} / \mathrm{m})^{\mathrm{a}}$ & $29.9(5.14)$ \\
\hline \multicolumn{2}{|l|}{ Sex, $N(\%)$} \\
\hline Female & $4,024(63.0)$ \\
\hline \multicolumn{2}{|l|}{ Race, $N(\%)^{\mathrm{b}}$} \\
\hline White & $5,325(83.4)$ \\
\hline Black & $866(13.6)$ \\
\hline Other & $1,96(3.1)$ \\
\hline Current smoker, $N(\%)$ & $636(10.0)$ \\
\hline Physically active, $N(\%)$ & $3,493(54.7)$ \\
\hline \multicolumn{2}{|l|}{ Handgrip tertile $^{\mathrm{b}} N(\%)$} \\
\hline Low & $1,473(23.1)$ \\
\hline Mid & $3,149(49.3)$ \\
\hline High & $1,766(27.7)$ \\
\hline Physical limitations (PL) & $3,803(59.5)$ \\
\hline Activities of daily living (ADL) & $1,217(28.2)$ \\
\hline Instrumental activities of daily living (IADL) & $2,404(38.5)$ \\
\hline
\end{tabular}

were run using PROC SURVEYLOGISTIC (SAS Institute Cary, NC). Models were adjusted for age (at time of physical assessment), years of education, ethnicity (white, black, and other), sex, smoking status (yes, no), body mass index (bmi) $\mathrm{kg} / \mathrm{m}^{2}$, and number of medical comorbidities and weighted using the HRS respondent-level weights for physical measures, which includes adjustments for sample selection probability and nonresponse. Detailed documentation regarding HRS sample weight calculations is available at http://hrsonline.isr.umich.edu/sitedocs/wghtdoc.pdf. All data was examined using statistical tests that were two-sided with $p$ values less than or equal to 0.05 . Confidence intervals excluding 1.0 were considered statistically significant.

\section{Results}

Sample characteristics are presented in Table 1. The sample was predominantly white $(83.4 \%)$ and female $(63 \%)$. Overall prevalence of PL, ADL, and IADL limitations were 3,803 (59.5\%), 1217 (28.2\%), and 2404 (38.5\%), respectively. Prevalence of PL, ADL, and IADL limitations was the highest in the lowest grip strength category, followed by MG, and was the lowest in the HG category. Prevalence of PL, ADL, and IADL limitations was also lower for those reporting engagement in physical activity within each grip category (Table 2).

Table 3 presents adjusted odds of PL, ADL, and IADL limitations by overall grip strength category and by activity level within each grip category. Overall odds of PL, ADL, and IADL limitation were significantly lower for those in MG category and the lowest in the HG category as compared to those in the LG grip category. In addition to increasing age, being female OR $2.00[1.66,2.42], p \leq .001$, BMI 1.08 [1.061.11], $p \leq .001$, number of comorbidities 1.71 [1.57-1.85], $p<$ .001 , and being a current smoker 1.80 [1.30-2.48], $p \leq .01$, were independently associated with higher odds of PL. These risk factors were also associated with increased odds of IADL limitations: OR 1.80 [1.49-2.19], $p<.001$ for female status, OR 1.04 [1.02-1.06], $p \leq .001$ for BMI, OR 1.72 [1.58-1.87], $p<.001$ number of comorbidities, and OR 1.95 [1.42-2.67], $p \leq .001$, for current smokers. For ADL limitations, race OR 1.39 [1.08, 1.78], $p<.01$, higher BMI OR 1.04 [1.02, 1.07], $p<.01$, and comorbidities OR $1.38[1.26,1.52], p \leq .001$, were associated with higher odds of limitation. Within grip categories, participating in physical activity did not appear to confer added benefits to reducing odds of disability or functional limitations.

\section{Discussion}

The current study examined the association between varying degrees of muscle strength, and odds of physical and functional limitations in older adults with central obesity who are physically active and inactive. We were primarily interested in (a) examining the prevalence of limitations by grip strength, (b) determining whether having higher grip strength was associated with reduced odds of physical and functional limitations, and (c) whether grip strength combined with PA is associated with additional reductions in physical and functional limitations. We found higher grip strength to be associated with lower prevalence of physical, ADL, and IADL limitations in elders with central obesity and increasing grip strength category to be significantly associated with progressively lower odds of physical, ADL, and IADL limitations after controlling for age, race, education, sex, BMI, medical comorbidities, and smoking status. This suggests that muscle strength is an important factor in preserving physical and functional impairment among those with central obesity independent of other known risk factors. Within grip categories, participating in physical activity was not associated with additional protection against odds of physical or functional limitations. In line with previous reports on risk factors for functional decline [25], we also found strong, independent associations between comorbidity and functional outcomes. These findings corroborate the need for targeted treatment and early intervention of chronic diseases among older adults, particularly the obese. Our findings extend the literature in that it provides supportive evidence to suggest that interventions aimed at improving muscle quality are beneficial even among obese elders with multiple health conditions.

Primary strengths of the current study include analyses of data from a nationally representative community dwelling sample of older adults with objective measures of grip strength and central adiposity (primary predictors). Limitations of the current analyses lie in the potential bias of self-reporting of physical outcomes and physical activity. 
TABLE 2: Prevalence of physical, ADL and IADL limitations by grip tertiles and physical activity.

\begin{tabular}{|c|c|c|c|c|c|c|c|c|c|}
\hline & \multicolumn{3}{|c|}{ Low grip } & \multicolumn{3}{|c|}{ Mid grip } & \multicolumn{3}{|c|}{ High grip } \\
\hline & Overall & Low PA & High PA & Overall & Low PA & High PA & Overall & Low PA & High PA \\
\hline Physical & $1126(76.4)$ & $698(84.8)$ & $428(65.9)$ & $1839(58.4)$ & $1002(70.9)$ & $837(48.2)$ & $838(47.5)$ & $395(59.9)$ & $443(40.0)$ \\
\hline $\mathrm{ADL}$ & $438(37.4)$ & $321(45.6)$ & $117(25.1)$ & $547(25.6)$ & $337(30.7)$ & $210(20.1)$ & $232(23.0)$ & $132(28.2)$ & $100(18.5)$ \\
\hline IADL & $826(57.6)$ & $556(69.3)$ & $270(42.8)$ & $1141(37.1)$ & 689 (49.6) & $452(26.7)$ & $437(25.2)$ & $237(36.1)$ & $200(18.6)$ \\
\hline
\end{tabular}

Note. Low grip $\leq 31.25$ men, $\leq 18.5$ women; high grip $\geq 43.5$ men, $\geq 26.5$ women. ADL limitations difficulty or inability with $\geq 1$ dressing, eating, or getting out of bed. IADL $\geq 1$ preparing meals, managing money, house/yard work.

TABLE 3: Odds of physical and ADL limitations by grip tertile and activity level.

\begin{tabular}{lccccccc}
\hline & $N$ & Overall & $\begin{array}{c}\text { Mid grip }^{\text {ab }} \\
\text { Low activity }\end{array}$ & High activity & Overall & $\begin{array}{c}\text { High grip }^{\text {ab }} \\
\text { Low activity }\end{array}$ \\
& & High activity \\
\hline Physical & 6,388 & $0.54(0.42,0.69)$ & $0.62(0.42,0.92)$ & $0.54(0.39,0.75)$ & $0.42(0.31,0.56)$ & $0.40(0.25,0.63)$ & $0.48(0.33,0.70)$ \\
ADL & 4,320 & $0.58(0.45,0.76)$ & $0.45(0.32,0.64)$ & $0.91(0.61,1.37)$ & $0.60(0.43,0.84)$ & $0.50(0.32,0.79)$ & $0.87(0.53,1.46)$ \\
IADL & 6,248 & $0.59(0.47,0.75)$ & $0.58(0.41,0.81)$ & $0.69(0.49,0.97)$ & $0.46(0.34,0.61)$ & $0.40(0.26,0.61)$ & $0.61(0.40,0.92)$ \\
\hline
\end{tabular}

Referent: low grip. ADL: activities of daily living, IADL: instrumental activities of daily living.

${ }^{a}$ Models are weighted and adjusted for age, sex, education, race, number of comorbidities, current smoking status, and body mass index.

${ }^{\mathrm{b}}$ Observations with zero or negative weights do not contribute to estimates and were automatically eliminated from the analyses $(n=3,028)$.

In addition, the cross-sectional nature of the data limits our ability to make causal inferences regarding the association between muscle strength, physical activity, and functional outcomes in elders with central adiposity. We also cannot speak to the long-term impact of muscle strength on physical and functional outcomes in obese older adults. Additional research is needed to determine whether muscle strength and physical activity are protective against long-term functional decline in older adults with central obesity in the presence of other chronic health conditions.

\section{Conclusion}

Our results provide evidence that muscle strength is important for reducing odds of physical and functional limitations in obese elders. Among obese elders who are unable to engage in traditional aerobic activity, interventions that focus on preserving muscle strength are a viable means of reducing physical and functional limitations.

\section{Appendix}

See Table 4.

\section{Conflict of Interests}

Cassandra M. Germain, Ph.D. is supported in part by NHLBI IRS Diversity Supplement no. 3R01HL109219-02S1. Elizabeth Vasquez, Ph.D., has no financial disclosures. John A. Batsis, M.D., is partially supported by UB4HP19206-0100 as a Medical Educator from the Health Resources and Services Administration, the Dartmouth Centers for Health and Aging, and has received support from the Department of Medicine, Dartmouth-Hitchcock Medical Center. He has served as a consultant to the legal firm Dinse, Knapp,
TABLE 4: Sample characteristics of missing grip.

\begin{tabular}{lc}
\hline$N$ & 163 \\
Age, years & $72.2(7.80)$ \\
Education, years & $11.22(3.36)$ \\
Comorbidities & $2.72(1.23)$ \\
Waist circumference $(\mathrm{cm})$ & $108.0(14.19)$ \\
Body mass index $(\mathrm{kg} / \mathrm{m})$ & $29.9(5.01)$ \\
Sex, $N(\%)$ & \\
$\quad$ Female & $142(89.0)$ \\
Race, $N(\%)$ & \\
$\quad$ White & $115(70.55)$ \\
$\quad$ Black & $36(22.09)$ \\
$\quad$ Other & $12(7.36)$ \\
Current smoker, $N(\%)$ & $20(12.27)$ \\
Physically active, $N(\%)$ & $56(34.366)$ \\
\hline
\end{tabular}

McAndrew LLC, for a clinical case review in 2012. There is no other conflict of interests to report.

\section{Authors' Contribution}

Cassandra M. Germain, Ph.D., contributed to conceptualization, data analyses, and writing. John A. Batsis, M.D., contributed to conceptualization and writing. Elizabeth Vasquez, Ph.D., contributed to conceptualization and data analysis. Douglas R. McQuoid contributed to the analysis and final review of the paper.

\section{Acknowledgments}

The authors would like to acknowledge Rebecca Namenek Brouwer, from Duke University, Office of Clinical Research, for careful review and editing of earlier versions of this paper. 


\section{References}

[1] Y. Wang and M. A. Beydoun, "The obesity epidemic in the United States-gender, age, socioeconomic, racial/ethnic, and geographic characteristics: a systematic review and metaregression analysis," Epidemiologic Reviews, vol. 29, no. 1, pp. 628, 2007.

[2] S. B. Angleman, T. B. Harris, and D. Melzer, "The role of waist circumference in predicting disability in periretirement age adults," International Journal of Obesity, vol. 30, no. 2, pp. 364373, 2006.

[3] L. Chaoyang, E. S. Ford, L. C. McGuire, and A. H. Mokdad, "Increasing trends in waist circumference and abdominal obesity among US adults," Obesity, vol. 15, no. 1, pp. 216-224, 2007.

[4] J. A. Batsis, C. M. Germain, E. Vásquez, F. Lopez-Jimenez, and S. J. Bartels, "Waist circumference, physical activity, and functional impairments in older U.S. adults: results from the NHANES 2005-2010," Journal of Aging and Physical Activity, vol. 23, no. 3, pp. 369-376, 2015.

[5] J. A. Batsis, A. J. Zbehlik, L. K. Barre, T. A. Mackenzie, and S. J. Bartels, "The impact of waist circumference on function and physical activity in older adults: longitudinal observational data from the osteoarthritis initiative," Nutrition Journal, vol. 13, no. 1, article 81, 2014.

[6] L. A. Schaap, A. Koster, and M. Visser, "Adiposity, muscle mass, and muscle strength in relation to functional decline in older persons," Epidemiologic Reviews, vol. 35, no. 1, pp. 51-65, 2013.

[7] V. L. Keevil, R. Luben, N. Dalzell et al., "Cross-sectional associations between different measures of obesity and muscle strength in men and women in a British cohort study," The Journal of Nutrition, Health \& Aging, vol. 19, no. 1, pp. 3-11, 2015.

[8] N. N. Hairi, R. G. Cumming, V. Naganathan et al., "Loss of muscle strength, mass (sarcopenia), and quality (specific force) and its relationship with functional limitation and physical disability: the concord health and ageing in men project," Journal of the American Geriatrics Society, vol. 58, no. 11, pp. 2055-2062, 2010.

[9] C. R. Gale, C. N. Martyn, C. Cooper, and A. A. Sayer, "Grip strength, body composition, and mortality," International Journal of Epidemiology, vol. 36, no. 1, pp. 228-235, 2007.

[10] S. Stenholm, D. Alley, S. Bandinelli et al., "The effect of obesity combined with low muscle strength on decline in mobility in older persons: results from the InCHIANTI Study," International Journal of Obesity, vol. 33, no. 6, pp. 635-644, 2009.

[11] M. E. Bowen, "The relationship between body weight, frailty, and the disablement process," The Journals of Gerontology, Series B: Psychological Sciences and Social Sciences, vol. 67, no. 5, pp. 618-626, 2012.

[12] M. E. Miller, W. J. Rejeski, B. A. Reboussin, T. R. Have, and W. H. Ettinger, "Physical activity, functional limitations, and disability in older adults," Journal of the American Geriatrics Society, vol. 48, no. 10, pp. 1264-1272, 2000.

[13] D. H. Paterson and D. E. R. Warburton, "Physical activity and functional limitations in older adults: a systematic review related to Canada's Physical Activity Guidelines," International Journal of Behavioral Nutrition and Physical Activity, vol. 7, article 38, 2010.

[14] E. H. Ip, T. Church, S. A. Marshall et al., "Physical activity increases gains in and prevents loss of physical function: results from the lifestyle interventions and independence for elders pilot study," Journals of Gerontology, Series A: Biological Sciences and Medical Sciences, vol. 68, no. 4, pp. 426-432, 2013.

[15] T. Rantanen, J. M. Guralnik, R. Sakari-Rantala et al., "Disability, physical activity, and muscle strength in older women: the women's health and aging study," Archives of Physical Medicine and Rehabilitation, vol. 80, no. 2, pp. 130-135, 1999.

[16] D. Kuh, E. J. Bassey, S. Butterworth, R. Hardy, and M. E. J. Wadsworth, "Grip strength, postural control, and functional leg power in a representative cohort of British men and women: associations with physical activity, health status, and socioeconomic conditions," Journals of Gerontology Series A: Biological Sciences and Medical Sciences, vol. 60, no. 2, pp. 224231, 2005.

[17] E. Vásquez, J. A. Batsis, C. M. Germain, and B. A. Shaw, "Impact of obesity and physical activity on functional outcomes in the elderly: data from NHANES 2005-2010," Journal of Aging and Health, vol. 26, no. 6, pp. 1032-1046, 2014.

[18] C. M. Germain, E. Vasquez, and J. A. Batsis, "Physical activity, central adiposity, and functional imitations in communitydwelling older adults," Journal of Geriatric Physical Therapy, In press.

[19] T. Rantanen, J. M. Guralnik, D. Foley et al., "Midlife hand grip strength as a predictor of old age disability," The Journal of the American Medical Association, vol. 281, no. 6, pp. 558-560, 1999.

[20] R. W. Bohannon, "Hand-grip dynamometry predicts future outcomes in aging adults," Journal of Geriatric Physical Therapy, vol. 31, no. 1, pp. 3-10, 2008.

[21] US Department of Health and Human Services: The Practical Guide to the Identification, Evaluation, and Treatment of Overweight and Obesity in Adult Bethesda: NIH-National Heart, Lung, and Blood Institute, 2000.

[22] M.-C. Pouliot, J.-P. Després, S. Lemieux et al., "Waist circumference and abdominal sagittal diameter: best simple anthropometric indexes of abdominal visceral adipose tissue accumulation and related cardiovascular risk in men and women," The American Journal of Cardiology, vol. 73, no. 7, pp. 460-468, 1994.

[23] A. M. Yorke, A. B. Curtis, M. Shoemaker, and E. Vangsnes, "Grip strength values stratified by age, gender, and chronic disease status in adults aged 50 years and older," Journal of Geriatric Physical Therapy, vol. 38, no. 3, pp. 115-121, 2015.

[24] D. Saliba, M. Orlando, N. S. Wenger, R. D. Hays, and L. Z. Rubenstein, "Identifying a short functional disability screen for older persons," The Journals of Gerontology, Series A: Biological Sciences and Medical Sciences, vol. 55, no. 12, pp. M750-M756, 2000.

[25] S. Stenholm, H. Westerlund, J. Head et al., "Comorbidity and functional trajectories from midlife to old age: the health and retirement study," The Journals of Gerontology A: Biological Sciences and Medical Sciences, vol. 70, no. 3, pp. 332-338, 2015. 


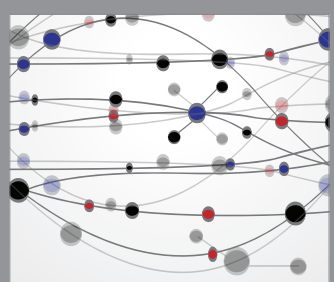

The Scientific World Journal
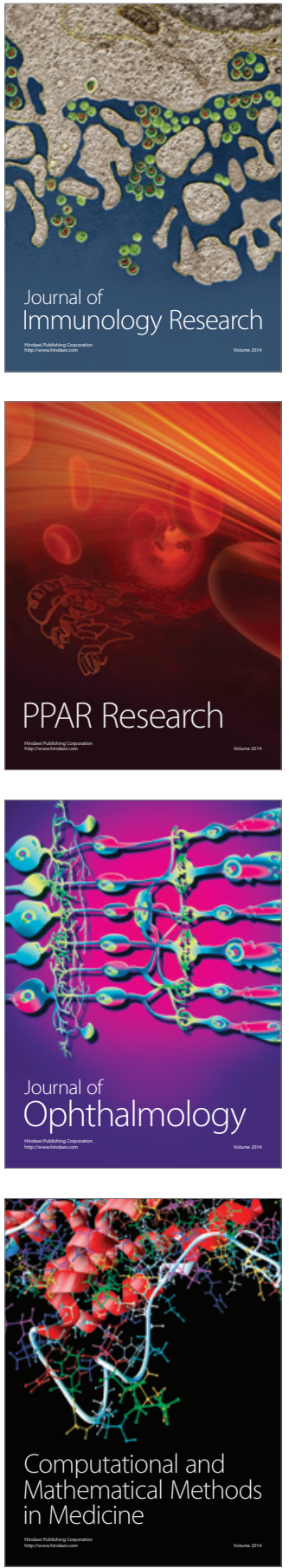

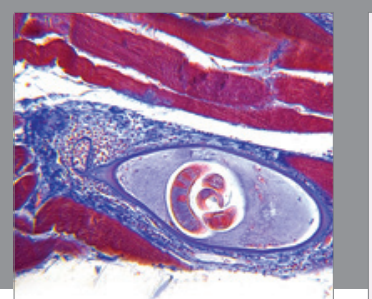

Gastroenterology Research and Practice

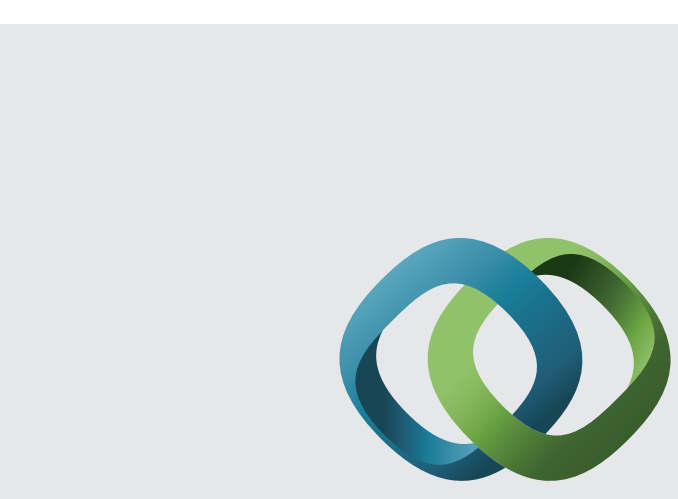

\section{Hindawi}

Submit your manuscripts at

http://www.hindawi.com
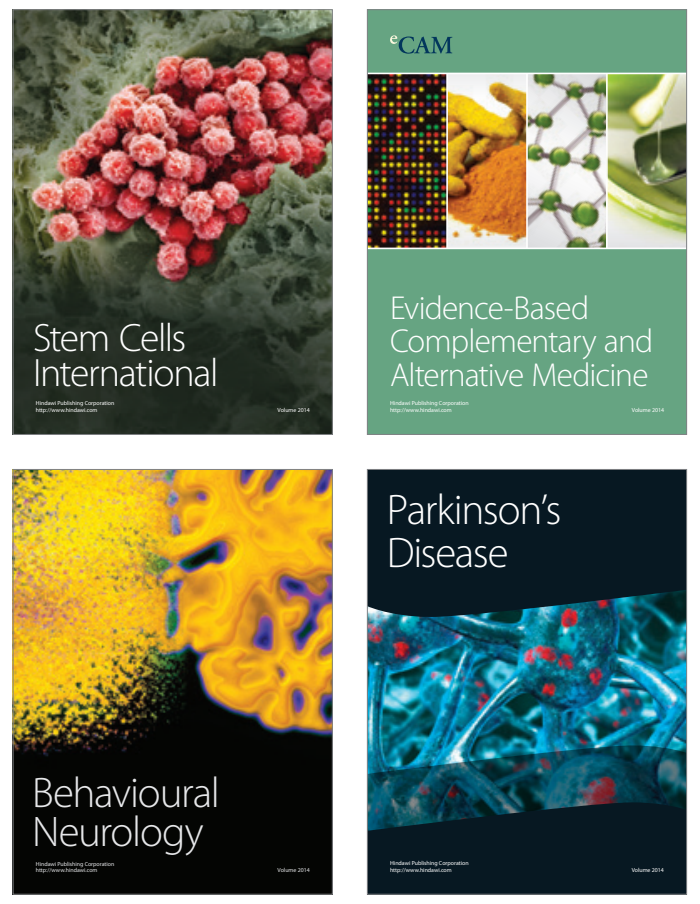
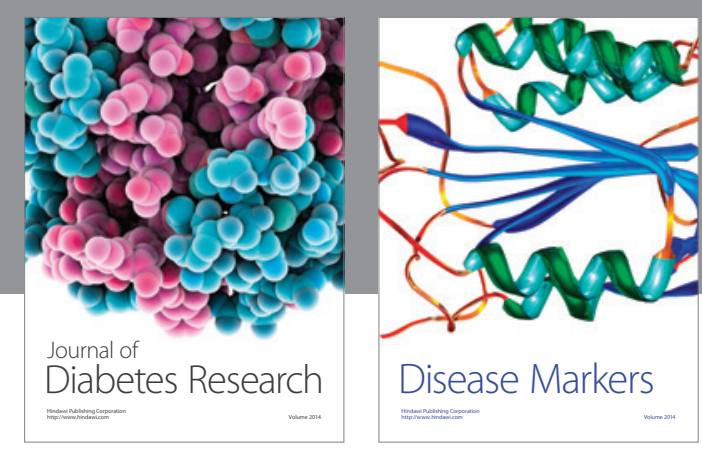

Disease Markers
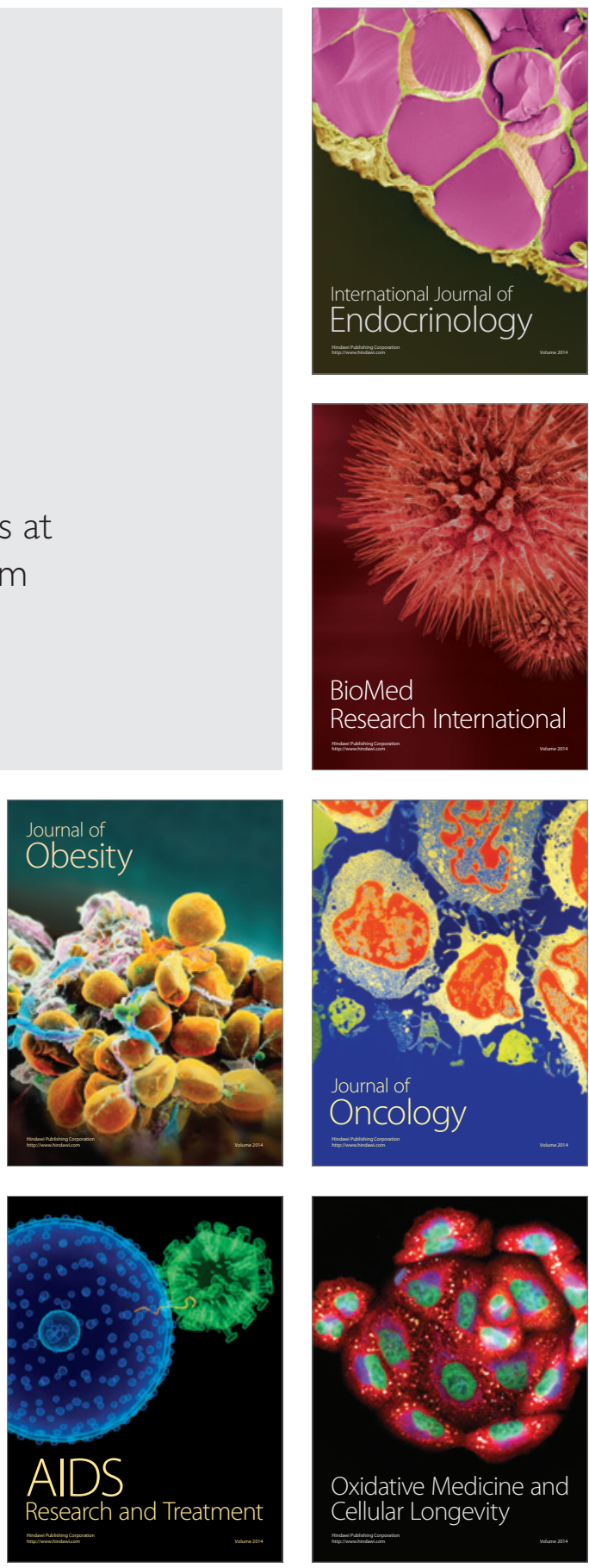